\title{
Qualidades Psicométricas da Escala de Atividade de Werry-Weiss-Peters: Estudo Preliminar da Versão Portuguesa
}

\author{
Psychometric Properties of the Werry-Weiss-Peters Activity Scale: Preliminar Study \\ of the Portuguese Version
}

\author{
Maria João Seabra-Santos ${ }^{1}$, Andreia Azevedo², Maria Filomena Gaspar ${ }^{3}$, Sofia Major ${ }^{4}$, Ana Sofia \\ Almeida $^{5}$ e Susana Eugénio ${ }^{5}$
}

\section{Resumo}

O rastreio e encaminhamento precoces de crianças em risco de Perturbação de Hiperatividade/ Défice de Atenção constituem desafios para médicos de cuidados primários, pediatras, psicólogos e pedopsiquiatras, interessados em interromper trajetórias de desenvolvimento desadaptativas. Este artigo analisa características psicométricas da versão portuguesa da Werry-Weiss-Peters Activity Scale (Routh, 1978), que avalia o nível de atividade de crianças em contexto familiar. Com base numa amostra normativa de 340 crianças (3-11 anos) e numa amostra de 157 crianças (3-6 anos) em risco de problemas de comportamento disruptivo, foi demonstrada a validade de uma pontuação única para o conjunto da escala, elevada consistência interna, estabilidade temporal e capacidade para discriminar entre crianças com e sem problemas de comportamento. Os resultados foram analisados em função do sexo e idade. A versão portuguesa da Escala WWP demonstrou bons índices de precisão e validade, sendo um instrumento de rastreio da hiperatividade potencialmente útil para profissionais e investigadores.

Palavras-chave: Werry-Weiss-Peters Activity Scale, rastreio, Perturbação de Hiperatividade/Défice de Atenção, propiedades psicométricas

\begin{abstract}
Early screening and referral of children at risk for Attention Deficit/ Hyperactivity Disorder are a challenge for primary care physicians, pediatricians, psychologists, and child psychiatrists aiming to interrupt deviant developmental trajectories. This paper analyses psychometric characteristics of the Portuguese version of the Werry-Weiss-Peters Activity Scale (Routh, 1978), that assesses children's activity level in family context. Based on a normative sample of 340 children aged 3 to 11, and a sample of 157 children aged 3 to 6 at risk for disruptive behavior problems, the validity of a single scale score was demonstrated, as well as high internal consistency, stability across time and capacity to discriminate between children with and without disruptive behavior. Results were analyzed according to children's gender and age. The Portuguese version of the WWP Scale shows evidence of good reliability and validity, and may therefore be a potentially useful screening measure of hyperactivity for professionals and researchers.
\end{abstract}

Keywords: Werry-Weiss-Peters Activity Scale, screening, Attention Deficit Hyperactivity Disorder, psychometric properties

Este trabalho foi parcialmente apoiado pela Fundação para a Ciência e Tecnologia e pelo FEDER-COMPETE através do projeto financiado PTDC/PSI-PED/102556/2008.

\footnotetext{
${ }^{1}$ Doutorada em Psicologia. Professora da Faculdade de Psicologia e de Ciências da Educação da Universidade de Coimbra, Portugal . Membro do Centro de Investigação do Núcleo de Estudos e Intervenção Cognitivo-Comportamental (CINEICC).

${ }^{2}$ Doutorada em Psicologia. Membro do Centro de Investigação do Núcleo de Estudos e Intervenção Cognitivo-Comportamental (CINEICC).

${ }^{3}$ Doutorada em Psicologia, Professora da Faculdade de Psicologia e de Ciências da Educação da Universidade de Coimbra, Portugal. Membro do Centro de Estudos Sociais da Universidade de Coimbra.

${ }^{4}$ Doutorada em Psicologia, membro do Centro de Estudos Sociais da Universidade de Coimbra, Portugal.

${ }^{5}$ Mestre em Psicologia pela Faculdade de Psicologia e de Ciências da Educação da Universidade de Coimbra, Portugal.

Correspondência: Rua do Colégio Novo, 3000-115 Coimbra Portugal. Tel.: +351 239851450. Fax: +351 239 851465. E-mail:

seabramj@fpce.uc.pt
} 


\section{Introdução}

A Perturbação de Hiperatividade/Défice de Atenção (PH/DA) representa uma das principais causas de encaminhamento para os serviços de saúde mental durante a infância (American Academy of Pediatrics [AAP], 2011), com uma prevalência mundial estimada, em amostras não clínicas, na ordem dos 5\% (Polanczyk, de Lima, Horta, Biederman, \& Rohde, 2007). Os sintomas, habitualmente diagnosticados em idade escolar, podem ocorrer mais cedo, durante os anos préescolares (Sonuga-Barke, Thompson, Abikoff, Klein, \& Brotman, 2006) ou mesmo aos 2 anos de idade (Egger \& Angold, 2006). Tomando em consideração a relevância do despiste e avaliação precoces na problemática em causa, e face à necessidade de medidas especificamente estudadas e validadas para a população portuguesa nas idades pré-escolar e escolar (Carapito, Ribeiro, \& Pereira, 2015; Major \& Seabra-Santos, 2014), o presente trabalho tem como objetivo proceder ao estudo das características psicométricas da versão Portuguesa da Escala de Atividade Werry-Weiss-Peters (WWP; Routh, 1978) numa amostra de crianças portuguesas entre os 3 e os 11 anos de idade. Pretende-se assim, proceder à adaptação desta escala, amplamente usada em estudos internacionais e valorizada pela facilidade do preenchimento e pelas boas características psicométricas, bem como testar a sua aplicabilidade e a pertinência da sua utilização por clínicos e por investigadores em Portugal.

A tendência para a manutenção dos sintomas de PH/DA ao longo do desenvolvimento (von Stauffenberg \& Campbell, 2007), a comorbilidade elevada dos sintomas com outros problemas, nomeadamente com a perturbação de oposição/desafio, o risco acrescido de problemas futuros, sobretudo quando os sintomas têm um início precoce (e.g., delinquência, baixo rendimento académico e abandono escolar, problemas interpessoais) (Daley, Jones, Hutchings, \& Thompson, 2009) e a interferência dos sintomas em diversos aspetos da qualidade de vida da criança (funcionamento físico, emocional, social e escolar) (Marques et al., 2013), assim como os elevados custos para a sociedade (Chorozoglou et al., 2015), têm levado os investigadores a impulsionar o estudo da PH/DA numa fase precoce do desenvolvimento (Dreyer, 2006). Assim, o despiste e intervenção atempadas, antes de os problemas se consolidarem e se associarem a outros, pode contribuir para uma trajetória desenvolvimental mais adaptativa (Sonuga-Barke \& Halperin, 2010). Este facto, aliado ao crescente número de crianças medicadas cada vez mais cedo (Zito et al., 2000) e às preocupações dos pais relativas aos efeitos da medicação a longo prazo, bem como à controvérsia em torno do uso de medicação em crianças tão novas (Sonuga-Barke et al., 2013), reforçam a necessidade de despiste e referenciação precoces, no sentido de implementar intervenções não farmacológicas, sempre que estas estejam disponíveis (Daley et al., 2009).

Neste âmbito, uma das principais questões da investigação prende-se com o diagnóstico precoce da $\mathrm{PH} / \mathrm{DA}$, que coloca desafios acrescidos ao clínico, devido ao risco de psicopatologização e sobrediagnóstico com base em problemas de desenvolvimento e de comportamento que podem ser transitórios. Os sintomas caraterísticos desta perturbação, isto é, a hiperatividade, a impulsividade e a desatenção fazem parte, em "doses aceitáveis", do desenvolvimento normativo das crianças, sobretudo em idades mais precoces (Brotman \& Gouley, 2008). Assim, e apesar da elevada variabilidade interindividual presente nestas faixas etárias, é importante estarmos atentos aos sinais da PH/DA e assegurar que as crianças são avaliadas de uma forma compreensiva, de acordo com uma perspetiva desenvolvimental e multidisciplinar (Bussing, Lehninger, \& Eyberg, 2006) em que o clínico deve recorrer a múltiplas fontes de informação (Daley et al., 2009) e a diferentes métodos de avaliação (entrevistas de diagnóstico, testes psicológicos, observação direta do comportamento). As escalas de avaliação preenchidas pelos pais e por outros informadores contam-se entre estes vários métodos, sendo uma das formas mais comuns de avaliação do nível de atividade da criança (Routh, Schroeder, \& O’Tuama, 1974).

\section{Escala de Atividade Werry-Weiss-Peters}

O principal objetivo da WWP é avaliar o nível de atividade da criança, de acordo com a perceção 
dos pais ou outros cuidadores do contexto familiar. Trata-se de uma escala de aplicação e cotação fáceis (McCann et al., 2014), que permite de uma forma breve e rápida despistar a presença e a severidade dos níveis de atividade da criança em diferentes situações da rotina diária (Barkley, Smith, Fisher, \& Navia, 2006). Este instrumento beneficia do facto de ter itens adaptados às características desenvolvimentais das crianças em diferentes níveis etários, nomeadamente em idade pré-escolar (Stormont, 2000). Originalmente concebida para avaliar os comportamentos em diferentes contextos (31 itens referentes a situações em casa e na escola; Werry, 1968), a Escala WWP foi posteriormente modificada e direcionada para a avaliação de comportamentos em contexto familiar (22 itens). O estudo da Escala foi retomado por Routh (Routh, 1978; Routh et al., 1974;), que chegou a uma versão composta por 27 itens que se combinam num resultado total único, versão esta que tem sido utilizada em inúmeras pesquisas internacionais (McCann et al., 2014; McGilloway et al., 2009; Perez, Turner, Fisher, Lockwood, \& Daley, 2014; Thompson et al., 2009). Essa é, igualmente, a versão que serve de base à presente pesquisa.

Os estudos psicométricos disponíveis revelam que a WWP é um instrumento válido, conseguindo discriminar entre crianças com e sem hiperatividade (Barkley, 1988) ou predizer eficazmente um diagnóstico clínico (SonugaBarke, Lamparelli, Stevenson, Thompson, \& Henry, 1994). Para além disso, noutros estudos o resultado da Escala WWP mostra correlacionar-se positivamente com outras medidas de hiperatividade preenchidas pelos pais, como a Escala de Conners (Barkley et al., 2006) e o Preschool Behaviour Questionnaire (PBQ; Campbell, Breaux, Ewing, \& Szumowski, 1986), bem como com medidas objetivas de atividade motora (Rapport, Kofler, \& Himmarich, 2006) ou medidas de atenção avaliadas através do computador (Harper \& Ottinger, 1992). Um estudo de Thompson e colaboradores (2009) aponta para a elevada estabilidade temporal do instrumento.

A WWP tem sido ao longo das últimas décadas largamente usada na avaliação dos efeitos de intervenções farmacológicas (Barkley, 1988) e de programas de intervenção parental, sobretudo Revista Iberoamericana de Diagnóstico y Evaluación - e Avaliação Psicológica. RIDEP · No44 · Vol.2 · 131-145 · 2017 em crianças com PH/DA (Azevedo, SeabraSantos, Gaspar, \& Homem, 2013; Azevedo, Seabra-Santos, Gaspar, \& Homem, 2015). Alguns estudos, utilizam a WWP também como medida de triagem dos sintomas de PH/DA (McCann et al., 2014; Perez et al., 2014; Thompson et al., 2009).

Em Portugal não temos conhecimento da existência de estudos de rastreio de sintomas de PH/DA com questionários desenvolvidos para a idade pré-escolar, apesar da existência de alguns estudos que utilizam questionários de "banda larga", que incluem na sua composição fatorial subescalas sensíveis à hiperatividade/problemas de atenção, como as Escalas de Comportamento para a Idade Pré-Escolar 2 (ECIP-2; Merrell, 2002; versão portuguesa de Major, 2011), o Inventário de Comportamento da Criança (CBCL 1.5-5; Achenbach \& Rescorla, 2000; Rescorla et al., 2012) e o Questionário de Capacidades e Dificuldades (SDQ; Goodman, 1997; versão portuguesa de Fleitlich, Loureiro, Fonseca, \& Gaspar, 2005), entre outros. Em idade escolar temos conhecimento da existência de dois estudos de prevalência da PH/DA com as Escalas de Conners (Conners, 1997) indicativos de uma taxa de $4.3 \%$ de crianças e adolescentes com sintomas de PH/DA na zona centro de Portugal Continental (Fonseca, Rebelo, Simões, \& Ferreira, 1995) e de $4.6 \%$ em crianças portuguesas de idade escolar (Melo, 2003). A escassez de estudos portugueses com instrumentos direcionados para as idades préescolar e escolar reforça a necessidade de investirmos nesta área e de tornar disponível para os clínicos e investigadores outras ferramentas de trabalho. Para além disso, sendo as escalas de avaliação comportamental a forma mais simples, rápida e económica de obter uma compreensão dos problemas da criança (Major, 2011), consideramos que a WWP pode ser uma maisvalia para a nossa população, em particular como meio auxiliar de diagnóstico numa fase inicial do processo de avaliação de uma problemática tão prevalente e multidimensional como a PH/DA. Neste trabalho, baseado em estudos exploratórios anteriores não publicados, é analisada a versão de 27 itens da Escala WWP de Routh (1978), nomeadamente quanto à respetiva estrutura fatorial, consistência interna, estabilidade 
temporal, validade convergente e validade baseada na diferenciação de grupos.

\section{Método}

\section{Participantes}

Foram incluídas neste estudo duas amostras: uma da população geral (normativa) e uma em risco de desenvolver problemas de comportamento do tipo disruptivo (doravante designada por "clínica"). A amostra normativa era composta por 340 crianças com 3 a 11 anos de idade $(M=5.83 ; D P=2.00)$, a frequentarem escolas escolhidas por conveniência no distrito de Aveiro ( $51 \%$ em jardins de infância e $49 \%$ em escolas do $1^{\circ}$ ciclo). Destas crianças, 164 (48\%) eram meninas e 176 (52\%) meninos. Os questionários foram respondidos maioritariamente pelas respetivas mães (82\%), e nalguns casos pelos pais (14\%), por ambos os progenitores ou por outros adultos significativos (4\%). As mães que responderam aos questionários tinham idades compreendidas entre os 20 e os 53 anos $(M=35.51 ; D P=5.49)$ e um número médio de anos de escolaridade igual a $11.69(D P=4.43)$. Quanto aos pais que responderam as idades oscilavam entre os 27 e os 55 anos $(M=38.45 ; D P=6.18)$ e o número médio de anos de escolaridade era igual a $11.12 \quad(D P=4.68)$. Os progenitores eram maioritariamente casados ou viviam em união de facto $(78 \%)$. A maioria destas famílias era de nível socioeconómico (NSE) médio (46\%) ou baixo (40\%), havendo 14\% de NSE elevado (de acordo com uma adaptação da classificação de Almeida, 1988, baseada nas profissões e nível escolar dos pais).

A amostra clínica, composta por 157 crianças em idade pré-escolar $(M=4.15 ; D P=.89)$ provinha de um outro estudo cujo objetivo principal era avaliar a eficácia de um programa de educação parental em crianças de idade pré-escolar com problemas de comportamento do tipo disruptivo e em variáveis relativas à parentalidade. Todas as crianças apresentavam níveis elevados de comportamentos externalizantes, de acordo com as suas mães, acima dos pontos de corte para a população portuguesa na subescala de hiperatividade $(21 \%)$, na subescala de problemas de comportamento (33\%), ou em ambas as subescalas (46\%) do Questionário de Capacidades e Dificuldades (SDQ). Muitas destas crianças (42\%) tinham sido sinalizadas por pediatras, pedopsiquiatras ou psicólogos por apresentarem esses comportamentos. O número de meninos, $n=109(69 \%)$ era superior ao de meninas, $n=48$ (31\%). A maioria dos questionários (98\%) foi respondida pelas mães, que eram maioritariamente casadas ou viviam em união de facto $(80 \%)$. As respondentes tinham uma média de idades de 36 anos $(D P=5.13)$ e um número médio de anos de escolaridade igual a $14.23(D P=3.72)$. A maioria destas famílias era de NSE médio (47\%), sendo outras de nível baixo $(28 \%)$ ou elevado $(25 \%)$.

\section{Instrumentos}

Questionáro Demográfico: Construído para o efeito com o objetivo de recolher as informações necessárias à caracterização da amostra.

Escala de Atividade de Werry-Weiss-Peters (Routh, 1978): Trata-se de um inventário de heterorresposta com 27 itens, que avalia o nível de atividade da criança em contexto familiar, em diversas situações do dia-a-dia (e.g., durante as refeições, a ver televisão, enquanto está a brincar, em atividades no exterior). A frequência com que certos comportamentos relacionados com o nível de atividade ocorrem é assinalada numa escala de tipo Likert que pode tomar os valores 0 ("Não ou raramente"), 1 ("Sim, algumas vezes") ou 2 ("Sim, muitas vezes"). O resultado total obtém-se somando diretamente as cotações dos itens e representa uma medida geral da atividade da criança (mínimo 0 e máximo 54). No final do inventário foram acrescentadas duas questões de resposta dicotómica (Sim/Não) relativas à ocorrência de acidentes (tal como uma queda) e à necessidade de a criança ser assistida num estabelecimento de saúde na sequência desse acidente.

Aos pais e aos educadores-deinfância/professores de um dos agrupamentos de escolas participantes $(n=216)$ foi pedido que classificassem os seus filhos/alunos em relação às três características que podem configurar um quadro de hiperatividade/défice de atenção (atividade excessiva, falta de atenção e impulsividade) em função do grau em que avaliavam tal característica como sendo 
problemática ("De modo algum"; "Problema Ligeiro"; "Problema Moderado"; "Problema Grave"). Para cada um destes parâmetros foi apresentada uma descrição operacional tendo em conta os critérios do DSM-IV-TR (APA, 2000/2002). A partir das respostas a estas perguntas foi calculado um resultado compósito para os pais e outro para os educadores-deinfância/professores (doravante designados genericamente por "professores").

\section{Procedimentos de tradução da Escala e de recolha dos dados}

A Escala de Atividade WWP foi traduzida para português por dois profissionais com bom domínio da língua inglesa, sendo um deles especializado em Psicologia Clínica e o outro em Avaliação Psicológica. Os itens em português foram retrotraduzidos para inglês por uma tradutora profissional. A comparação entre a versão inicial e a de chegada mostrou uma boa sobreposição no conteúdo dos itens.

A participação no estudo foi voluntária e anónima, tendo sido tomados em consideração os princípios éticos da investigação em Psicologia. Para a recolha da amostra normativa foi dirigido um pedido formal às direções dos jardins-deinfância e escolas participantes, através de uma carta onde foram apresentados os objetivos da investigação e realçada a confidencialidade dos dados recolhidos. Após a aprovação pelas direções os pais foram contactados através de uma carta onde se esclareciam os objetivos da investigação e se solicitava o seu consentimento informado por escrito. No mesmo envelope seguiam os questionários do estudo. A comunicação entre a equipa de investigação e os pais foi feita através dos professores, aos quais foram, igualmente, explicados os objetivos da investigação. Os pais que se disponibilizaram a participar (cerca de 56\% do total de pais abordados) devolveram os questionários preenchidos, novamente através de um envelope enviado junto com os questionários. Não foram analisados protocolos que apresentavam itens por responder (cerca de 5\% dos questionários preenchidos). Numa segunda fase, foi solicitada a colaboração dos pais de duas das turmas, selecionadas aleatoriamente, para voltarem a responder ao questionário (estudo de estabilidade Revista Iberoamericana de Diagnóstico y Evaluación - e Avaliação Psicológica. RIDEP · No44 · Vol.2 · 131-145 · 2017 temporal). Dos 42 questionários enviados nesta fase, 35 foram respondidos, o que corresponde a uma taxa de resposta de $83 \%$. Os procedimentos de recrutamento e recolha de dados na amostra clínica encontram-se descritos noutras publicações (Seabra-Santos et al., 2014, 2016).

\section{Tratamento estatístico dos dados}

O tratamento estatístico dos dados foi feito com recurso ao IBM SPSS Amos Version 20 para efetuar estudos de análise fatorial confirmatória (AFC), através do método de estimação de máxima verosimilhança. A fim de confirmar a estabilidade do modelo obtido, as duas amostras foram analisadas em conjunto $(N=497)$ para aumentar o leque de resultados e o poder da análise (Psychogiou, Daley, Thompson, \& Sonuga-Barke, 2008), e esta foi repetida separando as duas amostras: amostra normativa/clínica. Num primeiro momento, com base na literatura disponível (e.g., McGilloway et al., 2009; Thompson et al., 2009) os estudos foram realizados com os 27 itens da WWP com um único fator. De seguida, na tentativa de ultrapassar a menor fiabilidade dos itens trabalhados individualmente em estudos de AFC (Little, Cunningham, Shahar, \& Widaman, 2002), os 27 itens foram repartidos por seis parcelas atendendo ao tipo de atividade: Refeições (WWP_REF; itens 1-5), Ver televisão (WWP_TV; itens 6-10), Desenhar (WWP_DES; itens 11-15), Brincar (WWP_BRINC; itens 1620), Sono (WWP_SONO; itens 21-23) e Fora de Casa (WWP_FORA; itens 24-27). Foram considerados os seguintes índices de ajustamento do modelo: Chi-Square Goodness-of-Fit Test $\left(\chi^{2}\right)$, razão do $\chi^{2}$ pelos graus de liberdade $\left(\chi^{2} / d f\right)$, Goodness of Fit Index (GFI), Comparative Fit Index (CFI) e Root Mean Square Error of Approximation (RMSEA) com intervalo de confiança (IC) a 90\% (Jackson, Gillaspy, \& PurcStephenson, 2009; Marôco, 2010). Os valores de referência para avaliar o ajustamento do modelo considerados no presente estudo foram: $\chi^{2}$ com um valor reduzido associado a um nível de significância superior a $.05, \chi^{2} / d f$ inferior a 2 (Marôco, 2010), um ponto de corte próximo de .95 para o CFI e GFI e perto de .06 para o RMSEA (Hu \& Bentler, 1999). 
O programa IBM SPSS Statistics 20 foi utilizado para as restantes análises estatísticas. Nomeadamente, foram usadas estatísticas descritivas para a descrição das amostras e para caracterizar os resultados na Escala. A consistência interna foi avaliada através do coeficiente alfa de Cronbach e de correlações item-total. Foram calculados coeficientes de correlação de Pearson para estudar a estabilidade temporal da Escala de Atividade e a sua validade convergente com uma classificação de problemas de comportamento feita pelos pais e pelos professores. O teste $t$ de Student foi utilizado para fazer várias comparações entre médias. O efeito de algumas variáveis nos resultados foi explorado através de ANOVA ou do teste $t$ (para variáveis categoriais) e de correlações de Pearson (para variáveis contínuas).

\section{Resultados}

\section{Validade interna - Análise fatorial confirmatória}

Os estudos iniciais de AFC realizados com os 27 itens da WWP sugeriam um ajustamento pobre do modelo, $\chi^{2}(324)=1698.15, p<.001 ; \chi^{2} / d f=5.24$; $C F I=.77 ; G F I=.74 ; R M S E A=.09$. Atendendo ao conteúdo dos itens da WWP, foram realizados novos estudos de AFC com seis parcelas (cf. Figura 1). A análise dos valores da simetria $(S k)$ e curtose/achatamento $(K u)$ revelaram o cumprimento do pressuposto da normalidade ( $|S k|<3$ e $|K u|<10$; Marôco, 2010), apontando para um funcionamento adequado das parcelas (para as várias amostras: total, normativa e clínica).

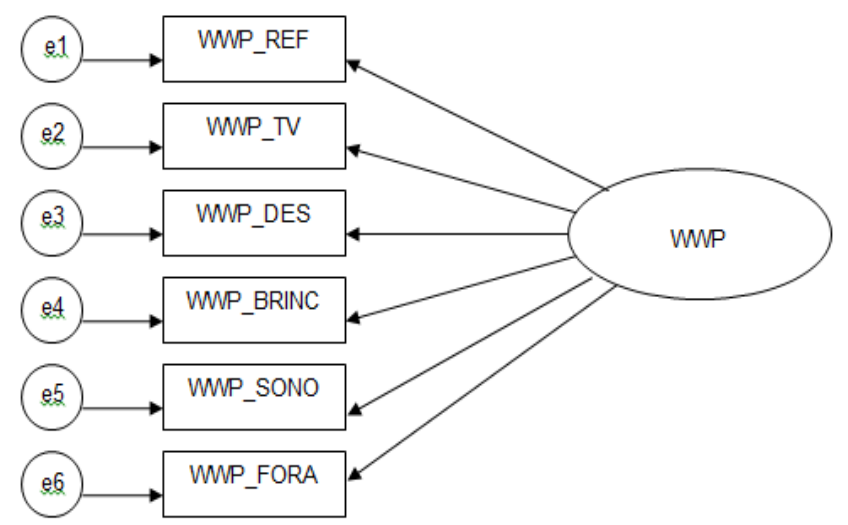

Figura 1. Representação esquemática AFC Escala de Atividade WWP
Os estudos de AFC com recurso às parcelas para a amostra total apontaram para cargas fatoriais estandardizadas com valores a oscilar entre .48 (WWP_SONO) e .86 (WWP_BRINC), sugerindo um bom ajustamento do modelo para a maioria dos indicadores considerados $\chi^{2}(9)=37.37, \quad p<.001 ; \quad \chi^{2} / d f=4.15 ; \quad C F I=.98 ;$ GFI=.98; RMSEA=.08 (IC 90\%=.05-.11). Ao replicar as análises desdobrando a amostra (normativa/clínica), o modelo de seis parcelas para a WWP revelou mais uma vez um bom ajustamento para a amostra normativa $\chi^{2}(9)=33.43, \quad p<.001 ; \quad \chi^{2} / d f=3.72 ; \quad C F I=.97 ;$ $G F I=.97 ; R M S E A=.09$ (IC 90\%=.06-.12); e clínica - $\chi^{2}(9)=15.82, \quad p<.001 ; \quad \chi^{2} / d f=1.76 ; \quad C F I=.97 ;$ GFI=.97; RMSEA $=.07$ (IC 90\%=.00-.13). As cargas fatoriais das parcelas situaram-se entre .43 (WWP_SONO) e .83 (WWP_BRINC) para a amostra normativa, e entre .19 (WWP_SONO) e .82 (WWP_BRINC) para a amostra clínica.

\section{Consistência interna}

Para a consistência interna da WWP foram obtidos valores iguais a $.93, .91$ e .88 , respetivamente para as amostras total $(N=497)$, normativa $(n=340)$ e clínica $(n=157)$. Apesar de ligeiramente mais baixos (o que está relacionado com a redução do número de itens de 27 para 6), os valores alcançados para a consistência interna das seis parcelas da WWP foram iguais a $.88, .85$ e .77 , respetivamente para as amostras total, normativa e clínica.

A validade interna dos itens foi avaliada através das correlações entre cada item e o total corrigido (retirado o item) e da contribuição de cada um deles para a consistência interna da Escala. Como os valores de alfa são elevados para as duas subamostras em estudo, são apresentados estes resultados somente para a amostra total, por razões de economia de espaço (Quadro 1). Como se pode verificar, todos os itens mostraram correlações com o resultado total superiores a 30 . $\mathrm{Na}$ sua maioria as correlações são, mesmo, superiores a .40 , havendo somente um item (o 22) que não respeita este critério mais exigente. Analisando as duas amostras separadamente, as correlações item-total mantiveram-se todas acima de .30 , exceto para os itens 21 e 22 na amostra clínica (valores iguais a $\quad .19$ e $\quad$ a $\quad .14$, respetivamente). 


\section{Estabilidade temporal}

A subamostra normativa que serviu de base ao estudo de estabilidade temporal era composta por 34 crianças a frequentarem o $1^{\circ}$ e $2^{\circ}$ anos de escolaridade, com 6 e 7 anos, $47 \%$ meninos e $53 \%$

Quadro 1. Estatísticas descritivas dos itens, correlação item-total corrigida e alfa se item retirado $(\mathrm{N}=497)$

\begin{tabular}{|c|c|c|c|c|}
\hline Item & $M$ & $D P$ & $\begin{array}{c}\text { Correlação } \\
\text { item-total } \\
\text { corrigida }\end{array}$ & $\begin{array}{l}\text { Alfa se } \\
\text { item } \\
\text { retirado }\end{array}$ \\
\hline 1 & 0.87 & 0.72 & .54 & .93 \\
\hline 2 & 0.91 & 0.65 & .56 & .93 \\
\hline 3 & 0.89 & 0.77 & .64 & .93 \\
\hline 4 & 0.78 & 0.78 & .62 & .93 \\
\hline 5 & 0.81 & 0.71 & .55 & .93 \\
\hline 6 & 0.73 & 0.71 & .58 & .93 \\
\hline 7 & 0.60 & 0.69 & .64 & .93 \\
\hline 8 & 0.66 & 0.70 & .56 & .93 \\
\hline 9 & 0.38 & 0.59 & .55 & .93 \\
\hline 10 & 0.52 & 0.67 & .62 & .93 \\
\hline 11 & 0.65 & 0.67 & .66 & .93 \\
\hline 12 & 0.55 & 0.66 & .67 & .93 \\
\hline 13 & 0.39 & 0.58 & .59 & .93 \\
\hline 14 & 0.54 & 0.66 & .62 & .93 \\
\hline 15 & 0.99 & 0.71 & .44 & .93 \\
\hline 16 & 0.61 & 0.72 & .64 & .93 \\
\hline 17 & 1.07 & 0.69 & .56 & .93 \\
\hline 18 & 0.99 & 0.70 & .52 & .93 \\
\hline 19 & 0.69 & 0.70 & .62 & .93 \\
\hline 20 & 0.28 & 0.52 & .50 & .93 \\
\hline 21 & 0.56 & 0.72 & .41 & .93 \\
\hline 22 & 0.29 & 0.59 & .34 & .93 \\
\hline 23 & 0.41 & 0.65 & .46 & .93 \\
\hline 24 & 0.46 & 0.63 & .49 & .93 \\
\hline 25 & 0.76 & 0.78 & .63 & .93 \\
\hline 26 & 0.63 & 0.69 & .65 & .93 \\
\hline 27 & 0.80 & 0.72 & .56 & .93 \\
\hline
\end{tabular}

meninas, sendo o intervalo de tempo médio entre as duas aplicações da Escala de Atividade de 25 dias.

O coeficiente obtido foi elevado, $r(30)=.93$, $p<.001$. A comparação entre as médias dos resultados obtidos nos dois momentos de avaliação, $\quad t(33)=1.40, \quad p>.05, \quad$ assinala $\quad$ a inexistência de diferença estatisticamente significativa entre os dois resultados.

\section{Validade convergente}

Foram calculados coeficientes de correlação de Pearson entre os resultados obtidos na WWP, preenchida por pais de crianças da amostra normativa, e dois resultados quantitativos compósitos obtidos a partir do modo como pais e professores, respetivamente, classificaram comportamentos da criança consonantes com os critérios de diagnóstico de PH/DA (atividade excessiva, impulsividade, falta de atenção). Embora em ambos os casos se tenham obtido coeficientes de correlação estatisticamente significativos, o valor foi mais elevado com o resultado obtido a partir das questões respondidas pelos pais, $r(213)=.70, \quad p<.01$, do que pelos professores, $r(214)=.32, p<.01$.

\section{Estatística descritiva}

Uma vez que o objetivo neste ponto era estabelecer uma base normativa preliminar para a Escala de Atividade WWP, recorreu-se somente à amostra normativa (Quadro 2), cujas diferenças de resultados em relação à amostra clínica foram já esclarecidas no ponto anterior. A análise dos resultados em função da idade mostrou existir uma diferença estatisticamente significativa entre os grupos etários, $F(6,326)=2.76, p<.05$ : os resultados tenderam a decrescer com a idade até aos 6 anos, o que coincide com a entrada na escola para a maioria das crianças, estabilizando a partir daí (com um valor médio mais baixo aos 8 anos). A análise em função do nível escolar, comparando as crianças pré-escolares com as que frequentam o $1^{\circ}$ ciclo, mostrou que as primeiras obtêm resultados significativamente mais elevados do que as segundas, $t(338)=3.20, p<.01$. Não se evidenciou diferença estatisticamente significativa entre as médias dos resultados em função do sexo, $t(338)=-0.11, p>.05$.

Os resultados totais apresentaram uma correlação negativa fraca mas significativa com a idade dos respondentes (pais), $\quad r(294)=-.17, \quad p<.01$, representando um efeito pequeno (Field, 2005), mas não se mostraram significativamente correlacionados com o número de anos de escolaridade completados por estes, $r(306)=-.03$, $p>.05$. 
Quadro 2. Estatísticas descritivas dos resultados para a amostra normativa total (N=340) e em função da idade, sexo e nível escolar das crianças

\begin{tabular}{|c|c|c|c|c|c|}
\hline & $n$ & Mínimo & Máximo & $M$ & $D P$ \\
\hline Amostra total & 340 & 0 & 48 & 13.35 & 8.78 \\
\hline \multicolumn{6}{|l|}{ Idade $^{(1)}$} \\
\hline 3 anos & 49 & 4 & 29 & 16.24 & 6.87 \\
\hline 4 anos & 52 & 0 & 41 & 14.44 & 8.82 \\
\hline 5 anos & 67 & 0 & 35 & 13.75 & 7.31 \\
\hline 6 anos & 45 & 0 & 34 & 12.00 & 7.88 \\
\hline 7 anos & 42 & 0 & 48 & 12.67 & 11.17 \\
\hline 8 anos & 42 & 1 & 32 & 9.76 & 7.37 \\
\hline 9 anos & 36 & 0 & 33 & 12.19 & 9.37 \\
\hline \multicolumn{6}{|l|}{ Sexo } \\
\hline Meninos & 176 & 0 & 48 & 13.30 & 8.84 \\
\hline Meninas & 164 & 0 & 44 & 13.40 & 8.75 \\
\hline \multicolumn{6}{|l|}{ Nível Escolar } \\
\hline Pré-escolar & 172 & 0 & 44 & 14.84 & 7.96 \\
\hline $1^{\circ} \mathrm{Ciclo}$ & 168 & 0 & 48 & 11.83 & 9.33 \\
\hline
\end{tabular}

O Quadro 3 apresenta valores de percentis, destacando-se o facto de estes diferirem em função do nível escolar das crianças. Assim, os percentis 80,85 e 90 correspondem a resultados na WWP mais elevados para as crianças préescolares, acontecendo o inverso para os percentis 95 e 98.

Quadro 3. Percentis para a amostra normativa total $(\mathrm{N}=340)$ e subamostras em função do nível escolar

\begin{tabular}{cccccc}
\hline & $\begin{array}{c}\text { Percentil } \\
80\end{array}$ & $\begin{array}{c}\text { Percentil } \\
85\end{array}$ & $\begin{array}{c}\text { Percentil } \\
90\end{array}$ & $\begin{array}{c}\text { Percentil } \\
95\end{array}$ & $\begin{array}{c}\text { Percentil } \\
98\end{array}$ \\
\hline $\begin{array}{c}\text { Amostra } \\
\text { total }\end{array}$ & 20 & 24 & 25 & 29 & 35 \\
$\begin{array}{c}\text { Pré- } \\
\text { escolar }\end{array}$ & 21 & 24 & 26 & 28 & 33 \\
$1^{\circ}$ Ciclo & 19 & 23 & 25 & 32 & 37 \\
\hline
\end{tabular}

\section{Validade de critério baseada na diferenciação de grupos}

Para analisar a capacidade da Escala de Atividade WWP para diferenciar o nível de atividade de crianças da população geral (amostra normativa) com o de crianças com características clínicas, compararam-se os resultados obtidos nas duas amostras anteriormente descritas. Porém, na amostra da população geral foram considerados somente os casos pré-escolares $(n=172)$, no sentido de equiparar as amostras quanto ao nível escolar e à idade, uma vez que a amostra clínica provinha de um outro estudo que visava somente crianças em idade pré-escolar $(n=157)$. As duas amostras não diferiam entre si nem quanto à idade das crianças nem dos respondentes, mas apresentavam diferenças significativas quanto ao número de anos de escolaridade completados pelos primeiros, $t(303)=-2.70, p<.01$, que era superior na amostra clínica $(M=14.23 ; D P=3.72)$ comparativamente à normativa $(M=13.01$; $D P=4.10)$. Em comparação com a amostra 
normativa, o número de meninas na amostra clínica era proporcionalmente inferior ao de meninos (1:1 e 1:2, respetivamente), $\chi^{2}(1$, $N=329)=10.04, \quad p<.01, \quad$ mas as amostras mostraram-se equivalentes quanto ao NSE, $\chi^{2}(2$, $N=321)=0.32, p>.05$.

A comparação entre as médias dos resultados obtidos na WWP pelas duas amostras revelou uma diferença estatisticamente significativa entre elas, $t(327)=-13.36, \quad p<.001, \quad$ com as crianças da amostra clínica a pontuarem mais elevado $(M=27.62 ; D P=9.39)$ que as da amostra normativa $(M=14.84 ; D P=7.96)$. Usando como referência o percentil 85 para o pré-escolar (21 pontos), chegou-se a um valor de sensibilidade igual a $77 \%$ e a um valor de especificidade igual a $79 \%$.

Para a amostra normativa foram, ainda, comparadas as médias dos resultados na WWP obtidos por crianças que tinham / não tinham recebido cuidados num centro de saúde ou num hospital na sequência dum acidente (por exemplo, resultante de uma queda). A diferença entre as médias na WWP revelou-se estatisticamente significativa, sendo as crianças do primeiro grupo avaliadas como mais ativas $(M=20.04 ; D P=11.92)$ do que as do segundo $(M=14.72 ; D P=10.17)$, $t(293.11)=-4.70, p<.001$.

\section{Discussão}

Esta pesquisa procurou validar a estrutura fatorial da versão portuguesa da Escala de Atividade WWP (Routh, 1978), confirmada pela adequação da maioria dos índices de ajustamento para o modelo (e.g., CFI, GFI) congruentes com os valores de referência apontados na literatura para abordagens mais exigentes ( $\mathrm{Hu} \&$ Bentler, 1999), e para as três amostras estudadas (total e desagregada em normativa/clínica).

Estes resultados vão ao encontro da opção de considerar um resultado total único na WWP para aglomerar os 27 itens que compõem esta Escala, opção esta igualmente seguida pelos autores da versão original e de outros estudos.

No que diz respeito à precisão da WWP, registam-se valores elevados do coeficiente alfa de Cronbach (Almeida \& Freire, 2003; Field, 2005), quer para a amostra total, quer para a amostra normativa e amostra clínica. Os vários itens apresentam correlações elevadas com o total, todas elas superiores ao limiar recomendável de .30 (Field, 2005; Pallant, 2005), sendo de assinalar, na amostra clínica, as exceções dos itens 21 (“A criança tem dificuldade em ir para cama?") e 22 (“A criança dorme pouco?”). Estes resultados são congruentes com os estudos de $\mathrm{AFC}$, em que a parcela WWP_SONO também apresentou menor peso fatorial nesta amostra clínica. Para estes dois itens, nesta amostra, os valores da correlação com o total são baixos, mesmo considerando formulações menos exigentes (valores $\geq .20$, tal como sugerido por Floyd \& Widaman, 1995). A análise do conteúdo destes dois itens e a comparação com a formulação original sugerem que, no caso do item 21, poderia ser mais pertinente focalizar a questão nas dificuldades que a criança possa ter em "permanecer na cama" (em vez de em "ir para a cama"). Independentemente desta consideração, os resultados sugerem que os itens relacionados com o deitar poderão ser menos relevantes na avaliação de crianças com problemas de comportamento de tipo disruptivo. É de assinalar a boa estabilidade temporal do instrumento, que constitui, igualmente, um bom indicador de fidedignidade.

As correlações estatisticamente significativas entre os resultados da WWP e a avaliação dos parâmetros comportamentais feita por pais e por professores são indicadores da validade convergente da Escala. $\mathrm{O}$ facto de a correlação ser superior quando o avaliador é o mesmo do que quando a informação é fornecida por dois avaliadores diferentes põe em destaque a variabilidade situacional do comportamento e as diferenças de perceção dos avaliadores, tal como evidenciado noutros estudos (Major, 2011; Seabra-Santos \& Almeida, 2014).

A análise dos resultados da amostra normativa em função de variáveis demográficas mostra não existirem diferenças em função do sexo. Este resultado deve ser considerado com precaução e alvo de averiguação posterior, uma vez que se desvia dos dados consistentemente reportados pela investigação nesta área, que aponta para uma maior incidência de comportamentos de tipo disruptivo em rapazes do que em raparigas (e.g., Major, 2011). Observa-se uma correlação negativa significativa, embora representando um efeito pequeno, entre os resultados na WWP e a $\mathrm{N}^{\circ} 44 \cdot \operatorname{Vol} .2 \cdot 131-145 \cdot 2017$ 
idade dos respondentes, a qual poderá ser explicada por uma maior confiança sentida pelos pais mais velhos quanto à sua capacidade para controlar o excesso de atividade dos seus filhos, ou uma maior tolerância relativamente ao comportamento dos mesmos. Por outro lado, pais mais velhos tenderão a ter filhos também mais velhos e, consequentemente, menos ativos. Investigações futuras poderão contribuir para explorar melhor esta associação.

Observa-se uma tendência para os resultados decrescerem com a idade até à entrada na escola, estabilizando a partir daí (tal como foi registado no estudo de Routh et al., 1974), o que explica que sejam mais elevados em crianças préescolares do que nas que frequentam o $1^{\circ}$ ciclo. Esta verificação deve ser compreendida em termos desenvolvimentais. Isto é, muitos dos comportamentos de atividade motora contemplados na WWP são normativos no caso de crianças muito novas (Brotman \& Gouley, 2008), assumindo características clínicas somente quando ultrapassam determinados limiares, e estes são diferentes em função da idade da criança. A análise dos percentis aponta-nos também para a necessidade de considerar referenciais normativos diferentes em função da idade, acrescentando dados potencialmente importantes em termos de diagnóstico e de intervenção precoce. Assim, as crianças de idade pré-escolar apresentam mais comportamentos relacionados com atividade excessiva quando consideramos um limiar de risco moderado (percentil 80). Porém, quando consideramos o limiar de risco grave (percentil 95) vemos que este se caracteriza por mais comportamentos disruptivos nas crianças em idade escolar (isto é, o grave é mais grave nestas idades). Por conseguinte, a sinalização atempada destas crianças é fundamental, se pensarmos na interferência negativa que tais comportamentos podem ter nas suas aprendizagens escolares e relações sociais, assim como no funcionamento da turma.

As crianças da amostra normativa foram reportadas na WWP como sendo significativamente menos ativas que as da amostra clínica. Apesar de as duas amostras não serem equivalentes quanto ao nível de escolaridade dos respondentes e ao sexo das crianças (sendo esta segunda divergência expectável atendendo à Revista Iberoamericana de Diagnóstico y Evaluación - e Avaliação Psicológica. RIDEP · No44 · Vol.2 · 131-145 2017 maior prevalência de problemas de comportamento em rapazes do que em raparigas), estas variáveis não mostram ter impacto sobre os resultados da WWP (cf. ponto dos resultados sobre estatística descritiva), pelo que as diferenças encontradas evidenciam a capacidade da Escala para diferenciar entre grupos com características reconhecidamente diferentes quanto ao nível de atividade (Barkley, 1988). Os valores obtidos para a sensibilidade $(77 \%)$ e para a especificidade (79\%) são equilibrados e aceitáveis para um instrumento deste tipo, podendo o valor da sensibilidade ser visto como uma estimativa por defeito do valor que se obteria recorrendo a uma amostra composta por crianças com um diagnóstico clínico formal (o que não acontece neste caso). Tais constatações constituem elementos favoráveis à validade da WWP enquanto questionário de rastreio de situações de risco para PH/DA, a qual também é colocada em evidência através dos resultados significativamente mais elevados registados em crianças da amostra normativa que foram assistidas medicamente na sequência de acidentes, por comparação com aquelas que não foram.

Este estudo comporta algumas limitações, nomeadamente relacionadas com a falta de representatividade da amostra normativa, recolhida por conveniência, e a não-equivalência entre a amostra normativa e a amostra clínica quanto a algumas variáveis demográficas. Estudos futuros poderão consolidar as conclusões da presente investigação recorrendo a uma amostra normativa de maiores dimensões, mais dispersa do ponto de vista geográfico e com uma melhor cobertura dos diferentes estratos socioeconómicos (cf. NSE elevado pouco representado na amostra do presente estudo). Em particular a análise sobre estabilidade temporal assentou numa subamostra de dimensões excessivamente reduzidas. Por outro lado, o facto de somente pouco mais de metade dos pais contactados terem aceite participar no estudo e terem efetivamente devolvido os questionários preenchidos, embora não seja inusual em estudos deste tipo, chama a atenção para a possibilidade de enviesamentos na seleção dos respondentes. No que diz respeito ao índices do ajustamento do modelo de AFC, alguns índices não alcançam os valores desejados. $\mathrm{O}$ valor do $\chi^{2} / d f$ fica ligeiramente acima do sugerido pela 
literatura, mas ainda assim é aceitável por ser inferior a 5, representando um "ajustamento sofrível" segundo os valores de referência propostos por Marôco (2010, p. 51). Quanto ao RMSEA (entre .07 e .09) os valores consideramse como aceitáveis (abaixo de .10), ainda que próximos do valor de .06 sugerido por $\mathrm{Hu}$ e Bentler (1999).

A presente investigação recorreu a questionários preenchidos quer por mães, quer por pais (estes em menor número). Uma amostra que comportasse um número idêntico de mães e de pais teria permitido compreender melhor as perceções de cada um dos progenitores sobre o nível de atividade das crianças, perceções estas que poderão não ser equivalentes (Berg-Nielsen, Solheim, Belsky, \& Wichstrom, 2012). Neste sentido, estudos futuros poderão examinar $\mathrm{o}$ acordo entre avaliadores e o modo como outras variáveis dos respondentes poderão ter impacto sobre os resultados. Análises mais finas, ao nível dos itens poderão, igualmente, contribuir para aumentar a utilidade do instrumento na identificação e caracterização de situações clínicas de atividade excessiva (Major \& Seabra-Santos, 2014). O desempenho académico e as aptidões sociais poderão constituir focos interessantes para outras investigações, no sentido de compreender o impacto que as características avaliadas pela escala WWP pode ter sobre o funcionamento e adaptação da criança à escola.

O presente estudo, apesar de preliminar, permitiu destacar algumas qualidades psicométricas de um questionário de triagem da hiperatividade para técnicos e investigadores, sensível às características das crianças em idade pré-escolar e escolar. A identificação precoce de situações de risco permitirá agir atempadamente no sentido de atenuar trajetórias desenvolvimentais desviantes e minimizar o impacto dos sintomas de PH/DA. Neste sentido, médicos de família, pediatras, psicólogos e pedopsiquiatras devem estar alerta para os primeiros sintomas de $\mathrm{PH} / \mathrm{DA}$, tendo presente que "intervir precocemente terá mais sucesso do que esperar até que os problemas estejam consolidados e depois tentar inverter o processo patogénico" (Sonuga-Barke \& Halperin, 2010, p. 370). Por conseguinte, o grande desafio reside no despiste e referenciação precoces. A WWP é um Revista Iberoamericana de Diagnóstico y Evaluación - e Avaliação Psicológica. RIDEP · No44 · Vol.2 · 131-145 · 2017 instrumento recomendável neste contexto, na medida em que combina a facilidade e rapidez de utilização com as boas características psicométricas demonstradas no presente artigo.

\section{Referências}

Abreu-Lima, I., Alarcão, M., Almeida, A., Brandão, T., Cruz, O., Gaspar, M., \& Santos, M. (2010). Avaliação de intervenções de educação parental: Relatório 2007-2010. Acedido de http://www.cnpcjr.pt/preview_doc umentos.asp? $\mathrm{r}=3493 \& \mathrm{~m}=\mathrm{PDF}$

Achenbach, T. M., \& Rescorla, L. (2000). Manual for the ASEBA preschool forms and profiles: An integrated system of multi-informant assessment. Burlington, VT: University of Vermont, Research Center for Children, Youth, and Families.

Almeida, L. (1988). O raciocínio diferencial dos jovens portugueses: Avaliação, desenvolvimento e diferenciação. Porto: Instituto Nacional de Investigação Científica.

Almeida, L. S., \& Freire, T. (2003). Metodologia da investigação em psicologia e educação ( $3^{\mathrm{a}}$ ed.). Braga: Psiquilibrios.

American Academy of Pediatrics (AAP). (2011). ADHD: Clinical practice guideline for the diagnosis, evaluation, and treatment of attention-deficit/hyperactivity disorder in children and adolescents. Pediatrics, 128(5), 1-16. doi: 10.1542/peds.2011-2654

American Psychiatric Association (2002). Manual de diagnóstico e estatística das perturbações mentais - Texto revisto ( $4^{\mathrm{a}}$ ed., J. N. Almeida, Trad.). Lisboa: Climepsi Editores. (Original publicado em 2000)

Azevedo, A. F., Seabra-Santos, M. J., Gaspar, M. F., \& Homem, T. C. (2013). The Incredible Years Basic Parent Training in Portuguese preschoolers at-risk for $\mathrm{AD} / \mathrm{HD}$ : Does it make a difference? Child and Youth Care Forum, 42, 403-424. doi: 10.1007/s10566013-9207-0

Azevedo, A. F., Seabra-Santos, M. J., Gaspar, M. F., \& Homem, T. C. (2015). Do Portuguese preschoolers with high hyperactivity behaviors make more progress than those with low hyperactivity after parental intervention? 
Journal of Early Intervention, 37(2), 119-137. doi: $10.1177 / 1053815115598006$

Barkley, R. A. (1988). Attention deficit disorder with hyperactivity. In E. J. Mash \& L. J. Terdal (Eds.), Behavioral assessment of childhood disorders (pp. 69-104). New York: Guilford.

Barkley, R., Smith, K., Fisher, M., \& Navia, B. (2006). An examination of the behavioral and neuropsychological correlates of three ADHD candidate gene polymorphisms (DRD4 7p, DBH TaqI A2, and DAT1 40 bp VNTR) in hyperactive and normal children followed to adulthood. American Journal of Medical Genetics Part B (Neuropsychiatric Genetics), 141B, 487-498. doi: 10.1002/ajmg.b.30326

Berg-Nielsen, T. S., Solheim, E., Belsky, J., \& Wichstrom, L. (2012). Preschoolers' psychosocial problems: In the eyes of the beholder? Adding teacher characteristics as determinants of discrepant parent-teacher reports. Child Psychiatry and Human Development, 43, 393-413. doi: 10.1007/s 10578-011-0271-0

Brotman, L. M., \& Gouley, K. (2008). Clinical assessment of preschoolers: Special precautions. In K. McBurnett \& L. Pfiffner (Eds.), Attention Deficit Hyperactivity Disorders: Concepts, controversies, new directions (pp. 235-242). New York: Informa Healthcare USA, Inc.

Bussing, R., Lehninger, F., \& Eyberg, S. (2006). Difficult child temperament and attentiondeficit/hyperactivity disorder in preschool children. Infants \& Young Children, 19(2), 123-131.

Campbell, S. B., Breaux. A., Ewing., L. J., \& Szumowski, E. K. (1986). Correlates and predictors of hyperactiviry and aggression: A longitudinal study of parent-referred problem preschoolers. Journal of Abnormal Child Psychology, 14, 217-234.

Carapito, E., Ribeiro, M. T., \& Pereira, A. I. (2015). Questionário de envolvimento parental no jardim-de-infância (QEPJI): Estudo de validação da versão para pais. Revista Iberoamericana de Diagnóstico y Evaluación -e Avaliação Psicológica. 40(1), 83-93.
Chorozoglou, M., Smith, E., Koerting, J., Thompson, M., Sayal, K., \& Sonuga-Barke, E. (2015). Preschool hyperactivity is associated with long-term economic burden: Evidence from a longitudinal health economic analysis of costs incurred across childhood, adolescence and young adulthood. Journal of Child Psychology and Psychiatry, 56(9), 966975. doi:10.1111/jcpp.12437

Conners, C. K. (1997). Conners' Rating Scales Revised. User's Manual. Toronto, ON: MultiHealth Systems, Inc.

Daley, D., Jones, K., Hutchings, J., \& Thompson, M. (2009). Attention deficit hyperactivity disorder in preschool children: Current findings, recommended interventions and future directions. Child: Care Health and Development, 35(6), 754-766. doi: 10.1111/j.1365-2214.2009.00938.x

Dreyer, B. P. (2006). The diagnosis and management of attention-deficit/hyperactivity disorder in preschool children: The state of our knowledge and practice. Current Problems in Paediatric Adolescent Health Care, 36, 6-30. doi: 10.1016/j.cppeds.2005.10 .001

Egger, H. E., \& Angold, A. (2006). Common emotional and behavioral disorders in preschool children: Presentation, nosology, and epidemiology. Journal of Child Psychology and Psychiatry, 47, 313-337. doi: 10.1111/j.1469-7610.2006.01618.x

Field, A. (2005). Discovering statistics using SPSS (and sex, drugs and rock ' $n$ ' roll) (3rd ed.). London: Sage.

Fleitlich, B., Loureiro, M., Fonseca, A., \& Gaspar, F. (2005). Questionário de capacidades e dificuldades (SDQ-Por). Acedido de www.sdqinfo.org.

Floyd, F. J., \& Widaman, K. F. (1995). Factor analysis in the development and refinement of clinical assessment instruments. Psychological Assessment, 7(3), 286-299. doi: 10.1037/1040-3590.7.3.286

Fonseca, A. C., Rebelo, J. A., Simões, A., \& Ferreira, J. A. (1995). Comportamentos antisociais no ensino básico: As dimensões do problema. Revista Portuguesa de Pedagogia, XXIX, 85-105. 
Goodman, R. (1997). The Strengths and Difficulties Questionnaire: A research note. Journal of Child Psychology, Psychiatry and Allied Disciplines, 38, 581-586. doi: 10.1111/j.1469-7610.1997.tb01545.x

Harper, G. W., \& Ottinger, D. R. (1992). The performance of hyperactive and control preschoolers on a new computerized measure of visual vigilance. The preschool vigilance task. Journal of Child Psychiatry and Psychology, 3(3), 1365-1372.

Hu, L., \& Bentler, P. M. (1999). Cutoff criteria for fit indexes in covariance structure analysis: Conventional criteria versus new alternatives. Structural Equation Modelling, 6(1), 1-55. doi: 10.1080/10705519909540118

Jackson, D. L., Gillaspy, J. A., \& PurcStephenson, R. (2009). Reporting practices in confirmatory factor analysis: An overview and some recommendations. Psychological Methods, 14(1), 6-23. doi: 10.1037/a0014694

Little, T. D., Cunningham, W. A., Shahar, G., \& Widaman, K. F. (2002). To parcel or not to parcel: Exploring the question, weighing the merits. Structural Equation Modeling, 9(2), 151-173. doi: 10.1207/S15328007SEM0902_ 1

Major, S. (2011). Avaliação de aptidões sociais e problemas de comportamento em idade préescolar: Retrato das crianças portuguesas. (Tese de Doutoramento). Acedido de https://estudogeral.sib.uc.pt/bitstream/10316/1 7774/5/ Tese_Sofia\%20Major.pdf

Major, S., \& Seabra-Santos, M. J. (2014). Aptidões sociais e problemas de comportamento: Retratos das crianças portuguesas de idade pré-escolar. Revista Iberoamericana de Diagnóstico y Evaluación - e Avaliação Psicológica, 38(2), 69-92.

Marôco, J. (2010). Análise de equações estruturais: Fundamentos teóricos, software \& aplicações. Pêro Pinheiro: ReportNumber.

Marques, J., Oliveira, J. A., Goulardins, J.B., Nascimento, R. O., Lima, A. M. V., \& Casella, E. B. (2013). Comparison of child self-reports and parent proxy-reports on quality of life of children with attention deficit hyperactivity disorder. Health and Quality of Life Outcomes, 11:186. doi: 10.1186/14777525-11-186
McCann, D. C., Thompson, M., Daley, D., Barton, J., Laver-Bradbury, C., Hutchings, J... Sonuga-Barke, E. (2014). Study protocol for a randomized controlled trial comparing the efficacy of a specialist and a generic parenting programme for the treatment of preschool ADHD. Trials, 15, 142-163. doi:10.1186/1745-6215-15-142

McGilloway, S. Bywater, T., Ni Mháille, G., Furlong, M., O'Neill, D., Comiskey, C.,..., Donnelly, M. (2009). Proving the power of positive parenting: A Randomised Control Trial to investigate the effectiveness of the Incredible Years Basic Parent training programme in an Irish context (short-term outcomes). Archways and NUI Maynooth. Acedido de http://www.archways.ie/fileadmin/user_uploa d/Files/Documents/Research_Report__Increidble_Years_Ireland_Study.pdf

Melo, A. I. R. (2003). Contributos para a avaliação da criança com Perturbação de Hiperactividade e Défice de Atenção (Tese de Doutoramento não publicada). Faculdade de Motricidade Humana da Universidade Técnica de Lisboa, Lisboa.

Merrell, K. W. (2002). Preschool and Kindergarten Behavior Scales - Second Edition. Austin, TX: PRO-ED.

Pallant, J. (2005). SPSS survival manual: A step by step guide to date analysis using SPSS version $12 \quad\left(2^{\text {nd }}\right.$ ed.). New York: Open University Press.

Perez, E., Turner, M., Fisher, A., Lockwood, J., \& Daley, D. (2014). Linguistic analysis of the preschool five minute speech sample: What the parents of preschool children with early signs of ADHD say and how they say It? PLoS ONE 9(9): e106231. doi:10.1371/journa .pone.0106231

Polanczyk, G., de Lima, M. S., Horta, B. L., Biederman, J., \& Rohde, L.A. (2007). The worldwide prevalence of ADHD: A systematic review and metaregression analysis. The American Journal of Psychiatry, 164, 942-948. doi: 10.1176/appi.ajp.164.6.94 2

Psychogiou, L., Daley, D. L., Thompson, M. J., \& Sonuga-Barke, E. J. S. (2008). Do maternal attention-deficit/hyperactivity disorder 
symptoms exacerbate or ameliorate the negative effect of child attentiondeficit/hyperactivity disorder symptoms on parenting? Development and Psychopathology, 20, 121-137. doi: 10.1017/S0954579408000060

Rapport, M., Kofler, M., \& Himmarich, C. (2006). Activity measurement. In M. Hersen (Ed.), Clinician's handbook of child behavioral assessment (pp. 125-157). Burlington, MA: Elsevier Academic Press.

Rescorla, L. A., Achenbach, T. M., Ivanova, M. Y., Bilenberg, N., Bjarnadottir, G., Denner, S... Verhulst, F. C. (2012). Behavioral/emotional problems of preschoolers: Caregiver/teacher reports from 15 societies. Journal of Emotional and Behavioral Disorders, 20(2), 68-81. doi: 10.1177/1063426611434158

Routh, D. (1978). Hyperactivity. In P. Magrab (Ed.), Psychological management of paediatric problems (pp. 3-8). Baltimore, MD: University Park Press.

Routh, D. K., Schroeder, C. S., \& O'Tuama, L. (1974). Development of activity level in children. Developmental Psychology, 10, 163168.

Seabra-Santos, M. J., \& Almeida, M. (2014). Falamos da mesma criança? Concordância mãe-pai-professores na avaliação do temperamento de crianças portuguesas. Psicologia: Reflexão \& Crítica, 27(1), 10-20.

Seabra-Santos, M. J., Gaspar, M. F., Azevedo, A., Homem, T. C., Guerra, J., Martins, V., ... Moura-Ramos, M. (2016). The Incredible Years parent training in Portuguese families: What changes, for whom, how, and for how long? Journal of Applied Developmental Psychology, 44, 93-104. doi:10.1016/j.appdev .2016 .04 .004

Seabra-Santos, M. J., Gaspar, M. F., Azevedo, A., Homem, T. C., Leitão, S., Pimentel, M., \& Major, S. (2014). Projeto "Prevenção/intervenção precoces em distúrbios de comportamento: Eficácia de programas parentais e escolares" - Protocolo de avaliação - Versão de Investigação. Acedido de http://fpce.uc.pt/anosincriveis/pro tocolo.doc
Sonuga-Barke, E., \& Halperin, J. (2010). Developmental phenotypes and causal pathways in attention/hyperactivity disorder: Potential targets for early intervention? Journal of Child Psychology and Psychiatry, 51(4), 368-389. doi: 10.1111/j.14697610.2009.02195.x

Sonuga-Barke, E., Brandeis, D., Cortese, S., Daley, D.,Ferrin, M., Holtmann, M.... European ADHD Guidelines Group (2013). Nonpharmacological interventions for ADHD: Systematic review and meta-analyses of randomized controlled trials of dietary and psychological treatments. American Journal of Psychiatry, 170, 275-289.

Sonuga-Barke, E., Lamparelli, M., Stevenson, J., Thompson, M., \& Henry, A. (1994). Preschool behaviour problems and intellectual attainment: The associations of hyperactivity and conduct problems. Journal of Child Psychology and Psychiatry, 35, 949-960. doi: 10.1111/j.1469-7610.1994.tb02304.x

Sonuga-Barke, E., Thompson, M., Abikoff, H., Klein, R., \& Brotman, L. M. (2006). Nonpharmacological interventions for preschoolers with ADHD: The case for specialized parent training. Infants and Young Children, 19(2), 142-153.

Stormont, M. (2000). Early child risk factors for externalizing and internalizing behaviors: a 5year follow-forward assessment. Journal of Early Intervention, 3, 180-190. doi: 10.1177/10538151000230030701

Thompson, M., Laver-Bradbury, C., Ayres, M., Le Poidevin, E., Mead, S., Dodds., C.... Sonuga-Barke, E. (2009). A small-scale randomized controlled trial of the revised New Forest parenting programs for preschoolers with Attention Deficit Hyperactivity Disorder. European Child and Adolescent Psychiatry, 18, 605-616. doi: 10.1007/s00787-009-0020-0

von Stauffenberg, C., \& Campbell, S. B. (2007). Predicting the early developmental course of symptoms of Attention Deficit Hyperactivity Disorder. Journal of Applied Developmental Psychology, 28, 536-552. doi: 10.1016/j.appdev.2007.06.011 
Werry, J. S. (1968). Developmental hyperactivity. Pediatric Clinics of North America, 15, 581599.

Zito, J. M., Safer, D. J., dosReis, S., Gardner, J. F., Boles, M., \& Lynch, F. (2000). Trends in the prescribing of psychotropic medications to preschoolers. JAMA, 283(8), 1025-1030. doi: 10.1001/jama.283.8.1025 